\title{
Significance of clearing differentiated thyroid carcinoma lymph node by high-frequency color Doppler ultrasonography
}

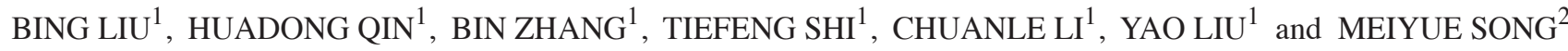 \\ Departments of ${ }^{1}$ General Surgery and ${ }^{2}$ Pathology, The Second Affiliated \\ Hospital of Harbin Medical University, Harbin, Heilongjiang 150001, P.R. China
}

Received April 21, 2016; Accepted November 23, 2016

DOI: $10.3892 / \mathrm{ol} .2016 .5450$

\begin{abstract}
We compared the clinical effects and prognosis of patients receiving lymph node dissection after surgical removal of the thyroid tissues and those not receiving it after the removal. A total of 80 patients diagnosed with differentiated thyroid carcinoma (DTC) by our hospital from March 2012 to March 2014 were successively included in the study. The cases were divided into the control group ( $\mathrm{n}=36$ cases) and observation group ( $n=44$ cases), and the two groups underwent total or subtotal resection of the thyroid. In the control group, patients underwent preoperative high-frequency color ultrasonography, and the most suspicious lymph node was removed. In the observation group, patients underwent preoperative high-frequency color ultrasonography, and the surgeons cleared the lymph node of the widest range. Difference in clinical effects and prognosis of the two groups were compared. After nearly a year's follow-up observation, the tumor recurrence rate of the observation group was significantly lower than that of the control group and the survival rate of the observation group was significantly higher than that of the control group $(\mathrm{P}<0.05)$. The rate of surgery complications and comparative difference of the two patient groups had no statistical significance $(\mathrm{P}>0.05)$. When comparing the data of lymphatic metastasis tested by preoperative high-frequency color ultrasonography with intraoperative diagnosed figures, sensitivity was $97.4 \%$, specificity $33.3 \%$, positive predictive value $90.2 \%$ and the negative predictive value $66.7 \%$. In conclusion, removal of the lymph node for DTC patients having undergone thyroid tissue excision with preoperative high-frequency color ultrasonography can be beneficial to improve the effects along with reduction in the recurrence rate.
\end{abstract}

Correspondence to: Dr Huadong Qin, Department of General Surgery, The Second Affiliated Hospital of Harbin Medical University, 157 Baojian Road, Harbin, Heilongjiang 150001, P.R. China

E-mail: huadong_qin1@163.com

Key words: differentiated thyroid cancer, lymph node dissection, high-frequency color ultrasonography

\section{Introduction}

The incidence of differentiated thyroid carcinoma (DTC) is very frequent in clinic, accounting for $90-95 \%$ of all thyroid carcinoma clinical incidence (1). With increasing number of cancer cases in the early stage of diagnosis, excision is becoming the first choice of treatment. Moreover, biological nature of differentiated thyroid cancer determines its low degree of malignancy and late lymphatic metastasis.

Total thyroidectomy and subtotal thyroidectomy are generally considered optimal options (2). However, some studies have suggested that based on lymph node partitions, the act of clearing the most suspicious lymph node does not increase the surgical complications, but reduces the possibility of tumor recurrence and second surgery (3).

In the present study, we studied the preoperative high-frequency color ultrasonography for the removal and range of the lymph nodes and obtained favorable results.

\section{Patients and methods}

Patients. In total 80 patients diagnosed with DTC at The Second Affiliated Hospital of Harbin Medical University (Heilongjiang, China) from March 2012 to March 2014 were successively included in the study. The patients suffered from hoarseness, drinking cough and diminishing physique of unknown origin or remained relatively asymptomatic. The patients were examined for thyroid nodule in their physical inspection, and were diagnosed with DTC after ultrasound guided fine needle puncture biopsy. The inclusion criteria for the study were: i) According to DTC diagnostic criteria; ii) age $\geq 18$ years, $<75$ years; and iii) no systemic metastasis. The exclusion criteria for the study were: i) Pregnancy; ii) combined hyperthyroidism, Hashimoto's thyroiditis and severe hypothyroidism; iii) patients with severe heart, liver, kidney and other organ dysfunction, or with mental disorder; and iv) patients with poor compliance or patients who refused to participate in the research.

After approval of the ethics committee of The Second Affiliated Hospital of Harbin Medical University and informed consent was obtained from patients or relatives, the cases were divided into the control group $(n=36$ cases) and observation group ( $\mathrm{n}=44$ cases). There were 14 male patients and 22 female patients in the control group, aged 37-66 years, 
with an average age of $46.9 \pm 12.3$ years. There were 32 cases of papillary carcinoma, and 4 cases of follicular carcinoma. The tumor diameter was $0.6-2.2 \mathrm{~cm}$, with an average of $1.4 \pm 0.3 \mathrm{~cm}$. There were 20 male patients and 24 female patients in the observation group, aged 35-68 years with an average of $48.7 \pm 13.4$ years. Furthermore, there were 39 cases of papillary carcinoma, 5 cases of follicular carcinoma, and the tumor diameter was $0.7-2.5 \mathrm{~cm}$ with an average of $1.6 \pm 0.5 \mathrm{~cm}$. A comparison of the differences of the gender, age, pathological type and tumor size showed no statistical significance $(\mathrm{P}>0.05)$.

Test methods. The patients in both groups underwent total thyroidectomy or subtotal thyroidectomy, the standard of which complied with the 2012 guideline for the diagnosis and treatment of thyroid cancer. Notably, attention was paid not to damage the parathyroid during surgery, and the combination of tracers e.g., methylene blue and nano carbon and micro-technique were applied if necessary. The surrounding tissue and structures were carefully separated and the recurrent laryngeal nerve was protected. However, in the control group, patients underwent preoperative high-frequency color ultrasonography, and the surgeons cleared the most suspicious lymph node according to experience. In the observation group, patients underwent preoperative high-frequency color ultrasonography, and the surgeons cleared the lymph node of the widest range. The difference in clinical effects and prognosis of the two groups were compared.

The ultrasound equipment used was Philips IU22 G4 high-frequency Doppler ultrasound with linear array wide-band probe, and the probe frequency was 7.5-10 MHz. Cervical lymph node metastasis standard diagnosed by ultrasound were (4): i) Change of aspect in ratio of lymph nodes $>1$; ii) irregular blood flow signals with lymph nodes; iii) irregular or blurred borders; iv) uneven internal echo; v) internal calcification; vi) unclear cortex and medulla boundaries; and vii) disappeared or cystic degeneration of hilar structure.

All the operations were performed by two Ultrasound experts. The ultrasound cervical lymph node partition referred to the dividing method of lymph nodes in the American Academy of Otolaryngology-Head and Neck. It was divided into seven areas (5): area I included submental area and submandibular lymph nodes, area II contained the internal jugular vein lymph node group lymph nodes, area III was middle jugular lymph nodes, area IV was lower jugular lymph nodes, area $\mathrm{V}$ was the carotid triangle jugular lymph nodes, area VI was anterior jugular lymph nodes (also known as central zone jugular lymph nodes), area VII was upper mediastinal lymph node (Figs. 1 and 2).

Observation index. The difference between the two groups was analyzed for tumor recurrence rate and survival rate as well as the rate of surgery complications after nearly a year's follow-up observation. After the comparison of the judgement of lymphatic metastases by preoperative high-frequency color ultrasonic results of definite diagnoses during surgery in the observation group, we obtained the sensitivity, specificity, positive predictive value and negative predictive value of ultrasonic diagnosis.

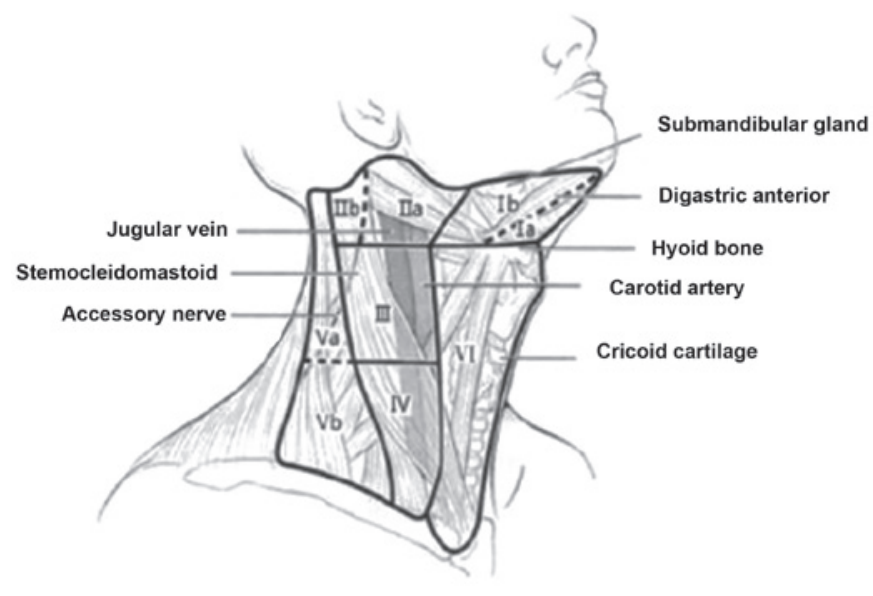

Figure 1. Cervical lymph node partition.

Statistical analysis. SPSS 20.0 statistical software (IBM SPSS, Armonk, NY, USA) was used for analysis and data was expressed as mean \pm standard deviation. Comparisons between groups were made using the t-test. The enumeration data were expressed as percentage (\%). Comparisons between groups were made using the $\chi^{2}$ test. $\mathrm{P}<0.05$ was considered to indicate a statistically significant difference.

Sensitivity was calculated as: True positive listings/(number of true-positive + number of false-negative) $\mathrm{x} 100 \%$. Specificity was calculated as: Number of true negative/(number of true-negative + number of false-positive) $\mathrm{x} 100 \%$.

Positive predictive value was calculated as the number of true positive/ (number of true-positive + false positive number) $\mathrm{x} 100 \%$, and the negative predictive value was calculated as the number of true negatives/(true-negative + number of false-negative number) $\times 100 \% . \mathrm{P}<0.05$ was considered statistically significant.

\section{Results}

Comparison of tumor recurrence rate and survival rate in the two groups. The tumor recurrence rate of the observation group was significantly lower than that of the control group and the survival rate of the observation group was significantly higher than that of the control group $(\mathrm{P}<0.05)$. The recurrence of tumor, and the time of death, of the two groups was not statistically significant $(\mathrm{P}>0.05$; Table I).

Comparison of the rate of surgery complications. The rate of surgery complications and comparative difference of the two patient groups was not statistically significant $(\mathrm{P}>0.05)$ as shown in Table II.

Comparison of the judgement of lymphatic metastases by preoperative high-frequency color ultrasonography with results of definite diagnoses during surgery in the observation group. A total of 44 patients were included in the observation group, 38 of whom were diagnosed with lymph node metastasis during surgery. The 34 cases of metastasis occurred in area VI (77.3\%), the number of metastasis was 1-4 with an average of $2.3 \pm 0.6$. The 2 cases of metastasis occurred in area III, 1 case occurred in area IV, and 1 case occurred in area II. Of the 
Table I. A comparison of tumor recurrence rate and survival rate in the two groups [n (\%)].

\begin{tabular}{lccccc}
\hline Group & No. of cases & Tumor recurrence rate & Recurrence time (month) & Survival rate & Death (month) \\
\hline Control & 36 & $11(30.6)$ & $10.5 \pm 2.3$ & $28(77.8)$ & $11.6 \pm 3.3$ \\
Observation & 44 & $5(11.4)$ & $11.6 \pm 3.4$ & $42(95.5)$ & $11.9 \pm 3.6$ \\
$\mathrm{t}\left(\chi^{2}\right)$ & & 4.558 & 0.624 & 0.156 & 0.925 \\
P-value & & 0.033 & 0.329 & 0.041 & 0.748 \\
\hline
\end{tabular}

Table II. Comparison of the rate of surgery complications [n (\%)].

\begin{tabular}{|c|c|c|c|c|c|c|}
\hline Group & No. of cases & $\begin{array}{l}\text { Parathyroid } \\
\text { injury }\end{array}$ & $\begin{array}{c}\text { Recurrent } \\
\text { laryngeal nerve }\end{array}$ & $\begin{array}{c}\text { Superior } \\
\text { laryngeal nerve }\end{array}$ & Others & $\begin{array}{r}\text { Rate of surgery } \\
\text { complications }\end{array}$ \\
\hline Control & 36 & 3 & 4 & 1 & 1 & $9(25.0)$ \\
\hline Observation & 44 & 5 & 5 & 1 & 2 & $13(29.5)$ \\
\hline $\mathrm{t}\left(\chi^{2}\right)$ & & & & & & 0.205 \\
\hline P-value & & & & & & 0.651 \\
\hline
\end{tabular}

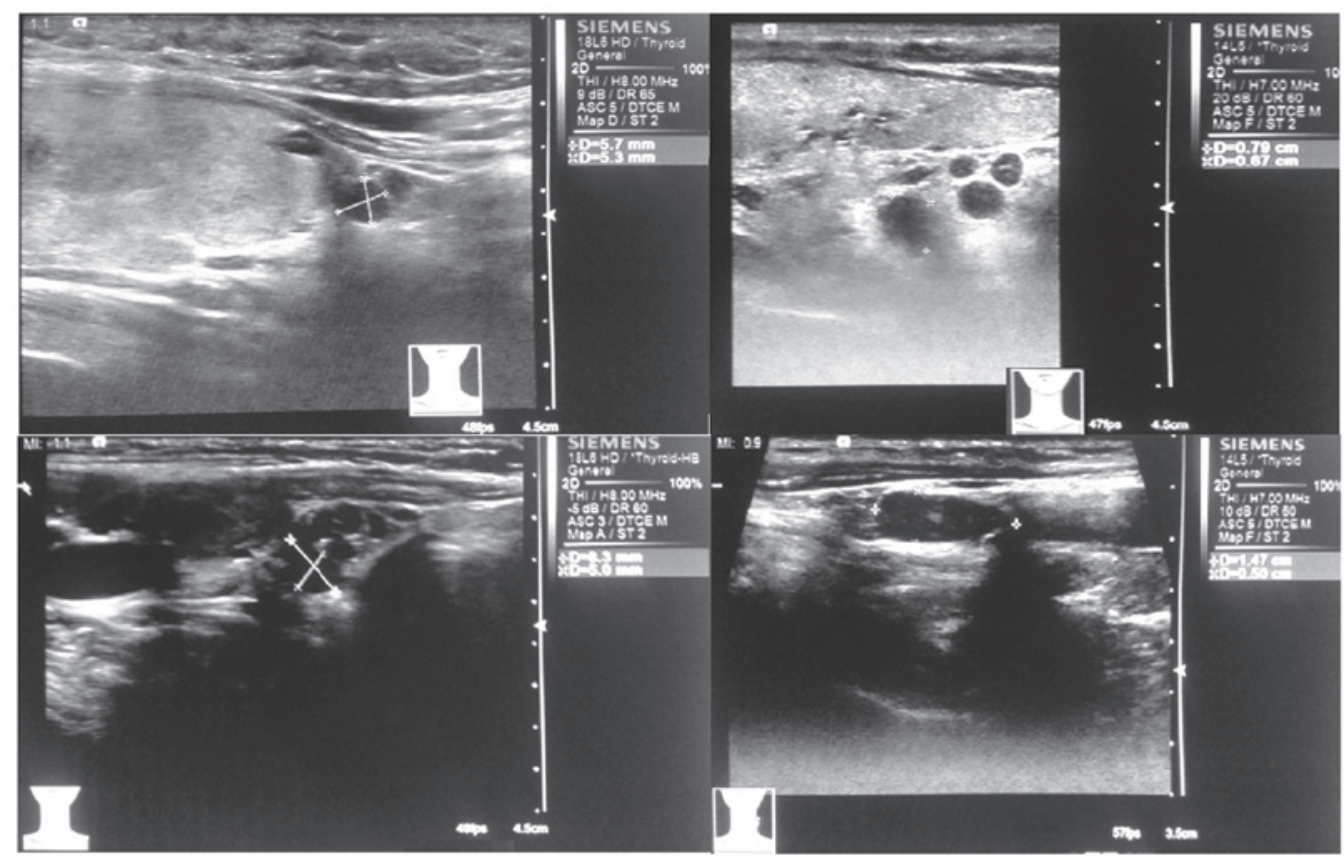

Figure 2. High frequency color Doppler ultrasonography for metastatic lymph nodes.

41 cases of suspicious lymph node removal found in preoperative ultrasound, 37 cases were true-positive, 4 cases were fasle-positive and 1 case was false-negative, 2 cases were true negative, the sensitivity was $97.4 \%$, specificity was $33.3 \%$, positive predictive value was $90.2 \%$ and negative predictive value was $66.7 \%$ (Table II).

\section{Discussion}

The basic principle of surgical treatment for cancer is that patients with lymph node metastasis need to undergo neck lymph node removal. However, the specificity of DTC biological behavior and complexity of neck dissection make the surgery highly risky, during which accidental injury and complications are likely to happen (6). There is no agreement on DTC neck lymph node removal, either at home or abroad. Studies have suggested that the enlarged neck lymph node removal range is much more likely to increase the risk of recurrent nerve and parathyroid injury rather than to have tumor recurrence, and the former leads to severe clinical complications and greatly lower patients' life quality (7). Nevertheless, it is undeniable that neck lymph node removal has become an important factor affecting 
the prognosis of thyroid cancer, the prognosis of patients is closely connected with primary surgical approach and surgical resection. The reasonable surgical approach can effectively reduce recurrence rate and reoperation rate (8).

Papillary thyroid carcinoma is the most typical tumor that mainly spreads to lymph node. Unilateral or bilateral carcinoma spreads to regional lymph nodes (9), and $20-90 \%$ patients with papillary carcinoma are detected with regional lymph node metastasis during diagnosis and the distant hematogenous metastasis only occurs in the later period (10). However, tumor of follicular thyroid mainly spreads through hematogenous metastasis. The rules and treating principle of neck lymph node metastasis are similar to that of papillary thyroid carcinoma (11). Central area (area VI) is the first station of lymph node metastasis manifested by paratracheal and pretracheal lymph node metastasis in ipsilateral neck central region (12). It is rare to have cases in which no metastasis occurs in the central area while saltatory metastasis to the lateral neck can be found under a microscope (13). However, when tumor spreads to lymph gland of lateral neck, lymph nodes in area II-V are considered to be infringed. Tumor generally spreads to multiple human regions rather than single region (14). With continuous application of high-frequency color ultrasonography and drawing on exprience, the accuracy of preoperative judgment of whether tumor metastasis occurs and the scope of metastasis is continuously improving (15). In the observation group, by comparing the judgement of lymphatic metastases by preoperative high-frequency color ultrasonic results of definite diagnoses during surgery, we found that the sensitivity was $97.4 \%$, specificity was $33.3 \%$, positive predictive value was $90.2 \%$ and negative predictive value was $66.7 \%$. To determine the criteria of neck lymph metastasis and neck lymph areas by color ultrasonography, there was a high degree of sensitivity and positive predictive value. Furthermore, ultrasonography is worthy of wide use due to its simple operations and reasonable price (16). The reason for insignificant specificity was connected to multiple factors, namely, the diameter of the primary tumor, the location of lymph node, patient's somatotype and the examiners' level of experience (17).

The study indicated that the tumor recurrence rate of the observation group was significantly lower than that of the control group and the survival rate of the observation group was significantly higher than that of the control group. The DTC spreads to lymph gland at a later time with low degree of malignancy and high excision rate and its prognosis is excellent. However, DTC is silent which leads to tumor recurrence and pro-metastasis of lymph node occurs in latter period after surgery. Moreover, surgical stimulation and disseminated lesions are risk factors for tumor recurrence (18). The study is not only concerned with papillary carcinoma but also follicular carcinoma, increasing the postive rate of results.

In comparison to previous studies, which only focused on papillary carcinoma with lower malignancy, the study has greater realistic significance. Some studies supported that follicular carcinoma and differentiated carcinoma with diameter more than $1 \mathrm{~cm}$ should undergo prophylactic lymphadenectomy (19). The comparison of surgery complications rate of two patient groups has no statistical significance. Currently, the application of tracers (e.g., methylene blue and nano carbon) and microtechniques can identify the parathyroid and lymph nodes, and retain the parathyroid tissue and blood supply provided complete excision in surgery, thus reducing the possibility of postoperative hypocalcemia symptoms (20).

In summary, to decide on removal and removal range of the lymph node for DTC patients having undergone thyroid tissue excision with preoperative high-frequency color ultrasonography can be highly accurate and beneficial to improving effects and reducing the recurrence rate, thus it is of great clinical significance.

\section{References}

1. Siegel R, Naishadham D and Jemal A: Cancer statistics, 2012. CA Cancer J Clin 62: 10-29, 2012.

2. Carty SE, Cooper DS, Doherty GM, Duh QY, Kloos RT, Mandel SJ, Randolph GW, Stack BC Jr, Steward DL, Terris DJ, et al; American Thyroid Association Surgery Working Group; American Association of Endocrine Surgeons; American Academy of Otolaryngology-Head and Neck Surgery; American Head and Neck Society: Consensus statement on the terminology and classification of central neck dissection for thyroid cancer. Thyroid 19: 1153-1158, 2009.

3. Cooper DS, Doherty GM, Haugen BR, Kloos RT, Lee SL, Mandel SJ, Mazzaferri EL, McIver B, Pacini F, Schlumberger M, et al; American Thyroid Association (ATA) Guidelines Taskforce on Thyroid Nodules and Differentiated Thyroid Cancer: Revised American Thyroid Association management guidelines for patients with thyroid nodules and differentiated thyroid cancer. Thyroid 19: 1167-1214, 2009.

4. Rotstein L: The role of lymphadenectomy in the management of papillary carcinoma of the thyroid. J Surg Oncol 99: 186-188, 2009.

5. Yüce I, Cağli S, Bayram A, Karasu F and Güney E: Regional metastatic pattern of papillary thyroid carcinoma. Eur Arch Otorhinolaryngol 267: 437-441, 2010.

6. Pisello F, Geraci G, Lo Nigro C, Li Volsi F, Modica G and Sciumè C: Neck node dissection in thyroid cancer. A review. G Chir 31: 112-118, 2010.

7. Vergez S, Sarini J, Percodani J, Serrano E and Caron P: Lymph node management in clinically node-negative patients with papillary thyroid carcinoma. Eur J Surg Oncol 36: 777-782, 2010.

8. Ferlito A, Robbins KT, Silver CE, Hasegawa Y and Rinaldo A: Classification of neck dissections: An evolving system. Auris Nasus Larynx 36: 127-134, 2009.

9. Hughes DT and Doherty GM: Central neck dissection for papillary thyroid cancer. Cancer Control 18: 83-88, 2011.

10. Moo TA and Fahey TJ III: Lymph node dissection in papillary thyroid carcinoma. Semin Nucl Med 41: 84-88, 2011.

11. Clark OH: Thyroid cancer and lymph node metastases. J Surg Oncol 103: 615-618, 2011

12. Zhang B, Niu HM, Wu Q, Zhou J, Jiang YX, Yang X, Li JC, Zhao RN, Wang M, Li KN, et al: Comparison of clinical and ultrasonographic features of poorly differentiated thyroid carcinoma and papillary thyroid carcinoma. Chin Med J (Engl) 129: 169-173, 2016.

13. Barbu CG, Florin A, Neamţu MC, Avramescu ET, Terzea D, Miron A, Dănciulescu Miulescu R, Poiană C and Fica S: Papillary thyroid carcinoma with anaplastic dedifferentiation in the lymph node metastasis - a rare form of presentation even for a tall cell variant. Rom J Morphol Embryol 56: 527-531, 2015.

14. Wang Q, Zhu X and Tan Z: The clinical significance of the Delphian lymph node metastasis in papillary thyroid carcinoma. Lin Chung Er Bi Yan Hou Tou Jing Wai Ke Za Zhi 29: 378-381, 2015 (In Chinese).

15. Liang XN, Guo RJ, Li S, Zheng ZM and Liang HD: Binary logistic regression analysis of solid thyroid nodules imaged by high-frequency ultrasonography, acoustic radiation force impulse, and contrast-enhanced ultrasonography. Eur Rev Med Pharmacol Sci 18: 3601-3610, 2014.

16. Ying M, Bhatia KS, Lee YP, Yuen HY and Ahuja AT: Review of ultrasonography of malignant neck nodes: Greyscale, Doppler, contrast enhancement and elastography. Cancer Imaging 13: 658-669, 2014. 
17. Isik S, Akbaba G, Berker D, Tutuncu YA, Ozuguz U, Aydin Y, Peksoy I and Guler S: Thyroid-related factors that influence preoperative localization of parathyroid adenomas. Endocr Pract 18: 26-33, 2012.

18. Lee CW, Gong G and Roh JL: Intraoperative diagnosis of central compartment lymph node metastasis predicts recurrence of patients with papillary thyroid carcinoma and clinically node-negative lateral neck and may guide extent of initial surgery. World J Surg 39: 194-202, 2015.
19. Li ZJ, An CM, Yan DG, Zhang XW, Zhang ZM, Xu ZG and Tang PZ: Significance of selective neck dissection in patients with cN0 thyroid carcinoma. Zhonghua Zhong Liu Za Zhi 35: 783-786, 2013 (In Chinese).

20. Chaojie Z, Shanshan L, Zhigong Z, Jie H, Shuwen X, Peizhi F, Jing X, Xiaowen G, Yang L and Wei Z: Evaluation of the clinical value of carbon nanoparticles as lymph node tracer in differentiated thyroid carcinoma requiring reoperation. Int J Clin Oncol 17: 32-34, 2015. 